\title{
Research on Feature Extraction Method of Engine Misfire Fault Based on Signal Sparse Decomposition
}

\author{
Canyi Du, ${ }^{1}$ Fei Jiang $\mathbb{D}^{2}{ }^{2}$ Kang Ding, ${ }^{2}$ Feng Li, ${ }^{1}$ and Feifei Yu ${ }^{1}$ \\ ${ }^{1}$ School of Automobile and Transportation Engineering, Guangdong Polytechnic Normal University, Guangzhou 510450, China \\ ${ }^{2}$ School of Mechanical and Automotive Engineering, South China University of Technology, Guangzhou 510640, China
}

Correspondence should be addressed to Fei Jiang; 201910100398@mail.scut.edu.cn

Received 22 December 2020; Revised 9 March 2021; Accepted 17 March 2021; Published 25 March 2021

Academic Editor: Jinde Zheng

Copyright (c) 2021 Canyi Du et al. This is an open access article distributed under the Creative Commons Attribution License, which permits unrestricted use, distribution, and reproduction in any medium, provided the original work is properly cited.

Engine vibration signals are easy to be interfered by other noise, causing feature signals that represent its operating status get submerged and further leading to difficulty in engine fault diagnosis. In addition, most of the signals utilized to verify the extraction method are derived from numerical simulation, which are far away from the real engine signals. To address these problems, this paper combines the priority of signal sparse decomposition and engine finite element model to research a novel feature extraction method for engine misfire diagnosis. Firstly, in order to highlight resonance regions related with impact features, the vibration signal is performed with a high-pass filter process. Secondly, the dictionary with clear physical meaning is constructed by the unit impulse function, whose parameters are associated with engine system modal characteristics. Afterwards, the signals that indicate the engine operating status are accurately reconstructed by segmental matching pursuit. Finally, a series of precise simulation signals originated from the engine dynamic finite element model, and experimental signals on the automotive engine are used to verify the proposed method's effectiveness and antinoise performance. Additionally, comparisons with wavelet decomposition further show the proposed method to be more reliable in engine misfire diagnosis.

\section{Introduction}

Because of its simple structure, output power stability, and convenient installation, the engine is widely utilized as the power output device in many mechanical systems, such as automotive, steamship, and spacecraft [1]. However, after the long-term harsh operation condition, the engine and other transmission system are prone to fault which will cause a sudden power interruption, thus affecting the reliability of the whole transmission system [2-4]. Furthermore, the vibration signals acquired from the sensor contain not only engine operating information but also various other strong noise signals, which causes useful feature signals to be submerged and makes it hard to diagnose engine fault. Therefore, it is of great significance to extract engine feature signals that indicate its health state and further monitor their operation status timely.

In terms of fault diagnosis in engine condition monitoring, many researchers have proposed various effective methods ranging from traditional signal processing to artificial intelligent algorithm. On the aspect of traditional signal processing methods, Bi et al. [5] proposed a novel fault diagnosis method of diesel engine valve clearance using the improved variational mode decomposition (VMD) and bispectrum algorithm. Vernekar et al. [6] applied empirical mode decomposition (EMD) to decompose the original vibration into a finite number of intrinsic mode functions and then used the Naïve Bayes algorithm as the classifier to detect the engine fault. Figlus et al. [7] applied a wavelet decomposition, while filtering the internal combustion engine's acoustic signal in order to diagnose an excessive valve clearance. On the aspect of diagnosis methods based on the artificial intelligent algorithm, it has been greatly developed and attracted more and more scholar's attention [8-11]. Deng et al. [8] proposed an adaptive method for choosing the parameters in the deep belief network (DBN) based on the improved quantum-inspired differential evolution algorithm, which could obtain higher classification 
accuracy on detecting rolling bearing fault. Du et al. [9] directly used the vibration signal as the input of the probabilistic neural network to simplify the diagnosis process and combined the swarm intelligent algorithm to achieve efficient parallel search for the best diagnosis effect. Jafarian et al. [10] employed various intelligent diagnosis methods, such as the Artificial Neural Networks (ANN) and Support Vector Machines (SVM), to monitor the engine health state and highlighted their superiorities. Tao et al. [11] proposed a novel extreme gradient boosting-based misfire fault diagnosis approach utilizing the high-accuracy time frequency information of vibration signals and achieved higher diagnosis accuracy. Although the above methods could achieve certain effects, most methods are easily influenced by other noises and cannot obtain good results under the low signalto-noise ratio (SNR).

Recently, due to its superiority of feature extraction ability under low SNR, sparse decomposition is widely applied in the field of image processing [12], compressed sensing [13], and fault diagnosis [14, 15]. Engine vibration signals have similar characteristics with these signals. Therefore, the sparse decomposition method can be theoretically transferred to the engine signal analysis and employed to extract its features for accurate diagnosis.

Additionally, like it is described in Reference [16], most of the reference simply used numerical simulation, which usually consists of sine and modulation components and noise part from the environment, to simulate the engine vibration signal and further to verify the proposed feature extraction method. However, the numerical simulation signal still has huge differences with the real-world complex engine signal. The algorithms validated by it may face trouble in handling the real engine vibration signal. Hence, to narrow the gap between the simulation signal and real signal as much as possible, a more concise dynamic finite element model should be constructed to generate the simulation signal rather than simply employ numerical simulation.

To address these problems, the proposed method combines the priority of signal sparse decomposition and engine finite element model to research a novel feature extraction method for engine misfire diagnosis in this paper. The organization of the rest paper is arranged as follows. Section 2 introduces the fundamental principle of the proposed method; Section 3 briefly illustrates the construction steps of the engine model and verifies the proposed feature extraction method; Section 4 is experimental verifications and comparison with wavelet analysis; Section 5 comes to conclusions.

\section{Feature Extraction of Engine Misfire Fault}

In the actual operating condition, the vibration signal collected from the hood is complex always contains unrelated signals generated by other components, which easily makes the feature signal represent the status of the engine submerged in strong noise. Additionally, different from other types of engine fault, misfire fault occurs with higher probability and its signal feature is relatively weak [17]. In order to detect engine misfire fault timely, it is necessary to find a method that can effectively extract its fault features under low SNR circumstances. Relying on its excellent feature extraction capabilities, the signal sparse decomposition method is therefore selected in this paper.

2.1. Basic Principle of MP. Matching pursuit (MP) is a sparse algorithm which utilizes linear combinations of atoms in the redundant dictionary to approximately represent a signal [14]. Due to its powerful signal sparse representation ability, $\mathrm{MP}$ is now widely applied in feature signal reconstruction to better detect imperceptible fault. Theoretically, the original signal $x$ can be decomposed into a linear superposition of atoms $\mathbf{d}(\|\mathbf{d}=1\|)$ in the dictionary $\mathbf{D} \in R^{n \times q}$, as shown in the following equation:

$$
x=\left|\left\langle x, \mathbf{d}_{0}\right\rangle\right| \mathbf{d}_{0}+R_{1} x,
$$

where $\mathbf{d}_{0}$ denotes the best matching atom in this procedure and $R_{1} x$ is the residual signal after first MP. Because of the orthogonality between $\mathbf{d}_{0}$ and $R_{1} x, R_{1} x$ can further decompose in the same way as $x$. After applying $N$ times to the residual signal, $x$ can be represented as follows:

$$
x=\sum_{n=0}^{N-1}\left|\left\langle R_{n} x, \mathbf{d}_{n}\right\rangle\right| \mathbf{d}_{n}+R_{N} x,
$$

In order to avoid falling into the infinite loop, the iterative procedure stops until $R_{N} x$ is less than a preset threshold $\delta$, which is a small value. From the above introduction, it can be known that the extraction accuracy of the feature signal mainly depends on the atoms in the dictionary. The more similar atoms with the feature signal, the better feature extraction result can be obtained.

2.2. Construction of the Dictionary with Physical Meaning. As we know, the unit response function has an attenuated oscillation waveform. The feature signals of engine misfire fault are continuous impact signals, which have a similar waveform with the unit response function in the time domain. Moreover, according to the principle of mechanical vibration, engine system's modes that are determined by modal parameters $\left(f_{d}, \xi\right)$ would be effectively excited by series of impulse forces generated from the cylinder. Furthermore, its parameters could influence structure physical vibration characteristics. The natural frequency $f_{d}$ and damping ratio $\xi$ determine the speed of vibration and attenuation in the time domain, respectively. And, these parameters are also connected with the physical structure of the engine system. Therefore, according to the similarity principle between the original signal and atom, it is reasonable to choose the unit response function as an atom in the sparse dictionary [14]. Its specific expression is presented as follows:

$$
d(t)=\exp \left(\frac{-2 \pi \xi}{\sqrt{1-\xi^{2}}} f_{d} t\right) \sin 2 \pi f_{d} t
$$


where $f_{d}$ and $\xi$ are the natural frequency and damping ratio, respectively. Equation (3) indicates that the atom is associated with the modal parameters of the engine again, which not only has high similarity with the original signal but also contains clear physical meaning.

2.3. Detailed Procedures of the Proposed Method. Inspired by $\mathrm{MP}$ and the engine vibration signal characteristics, the proposed method can be illustrated as shown in Figure 1. It mainly contains three parts: signal preprocessing, dictionary construction, and feature extraction. Due to the low-frequency noise caused by other machinery rotational components in the engine transmission system, the first part is utilized to improve the SNR of the obtained signal. The second is to construct a dictionary with clear physical meaning. Finally, the third is to apply MP to extract the engine feature signal for diagnosis. The detail procedures of the algorithm are implemented as follows:

(1) The signal $x(t)$ is obtained with a sampling frequency $f_{s}$ from the vibration acceleration sensor installed in the hood. Afterwards, a high-pass filtering process is applied to the collected signal to remove the low-frequency noise. This procedure can further improve the SNR of the signal and minimize interferences from other irrelevant signals as much as possible.

(2) According to the theory proposed in Reference [15], the multiorder frequencies and damping ratios $\left(f_{d j}, \xi_{j}\right)(j=1, \ldots, J)$ are obtained by edited cepstrum and rational fractional polynomial fitting method. Therefore, the atoms in the dictionary are constructed by equation (3). In order to expand the atoms and achieve the redundancy property of the dictionary, the time shift factor $\tau \in\left(0: \Delta t: T_{s}\right)$ is introduced to find out impact time of the signal. At last, the dictionary $\mathbf{D}$ consists of all the expanded atoms, with the shape of $p \times q . p=J \times \operatorname{round}\left(\left(T_{s}+\right.\right.$ 1)/ $\Delta t$ ) and $q=T_{s} f_{s}$, where $J$ is the maximum order of extracted modal parameters, $\Delta t$ represents the time shift interval, and $T_{s}$ is the ignition impact interval, and the notion of round presents for rounding off.

(3) To accelerate the calculation speed of MP, the signal is performed in $N$ segmental. Each segment of the filtered signal $x_{p}(t)$ is divided by a duration of $T_{s}$, which is corresponding to the ignition impact interval, namely, the reciprocal of main exciting frequency in the automotive engine. $T_{s}=\left(1 / f_{\text {main }}\right)=((60 \times 2) / n A)$, where $n$ is the rotation speed of the engine and $A$ is the amount of the engine cylinder.

(4) Set the iteration value $\delta$ of the MP algorithm, and apply it to solve sparse coefficients of the $n^{\text {th }}$ segment signal. Hence, the feature signal of the $n^{\text {th }}$ segment signal can be represented by $x_{f n}(t)$.

(5) Based on the dividing time sequential of the signal, the reconstructed feature signal of the engine can be expressed by equation (4). It should be mentioned that it is a quick algorithm to reconstruct the engine feature signal, and the times of inner product equals to round $\left(T / T_{s}\right) N p$, where $N$ is the maximal times of MP:

$$
x_{f}(t)=\sum_{n=1}^{N} x_{f n}(t) .
$$

(6) Finally, the feature of engine operating status could be represented by the reconstructed signal $x_{f}(t)$ and the misfire fault diagnosis is carried out.

\section{Simulation Analysis}

To verify the effectiveness of the proposed method, a precise engine finite element model is firstly applied to generate more realistic engine simulation signals. Then, white noises are added into the original signals to research its feature extraction ability under the low SNR condition. Comparison analysis illustrates the advantages of the proposed method in terms of feature extraction ability.

3.1. Generation of the Engine Simulation Signals. Different from other fault signals, such as the bearing fault signal or broken gear signal, the engine signals are more complex whether under the health state or misfire fault state. Therefore, in order to generate more realistic engine signals for analysis, a high-quality four-cylinder engine multibody dynamic model is constructed by utilizing the AVL EXCITE platform [18]. Its main procedures are as follows:

(1) Build the 3-dimensional model of the four-cylinder engine power unit and crankshaft in CAD software, and import it into MSC Patranto to perform finite element mesh division, as shown in Figure 2. This step is to set the engine as a flexible body and make each part of the vibration signal obtainable, especially the hood part.

(2) As we know, the dynamic system analysis with huge degrees of freedom is time consuming. Hence, the matrix reduction method is accomplished by MSC Nastran to reduce the degrees of freedom of the constructed model and then improve the calculation efficiency.

(3) Import the reduced finite element model into the EXCITE power unit, and each component can be connected by springs, dampers, bearings, and other nonlinear elements to form the final coupled engine dynamic model.

(4) Set combustion pressure in the EXCITE PU module as the external excitation to make the whole system vibrate. Its variation law along with the crank angle under the normal state is illustrated in Figure 3(a), which is similar to actual engine working conditions. Meanwhile, the engine misfire fault is simulated by setting the combustion pressure of the corresponding faulty cylinder into a low value, as shown in Figure 3(b). It should be mentioned that the crank 


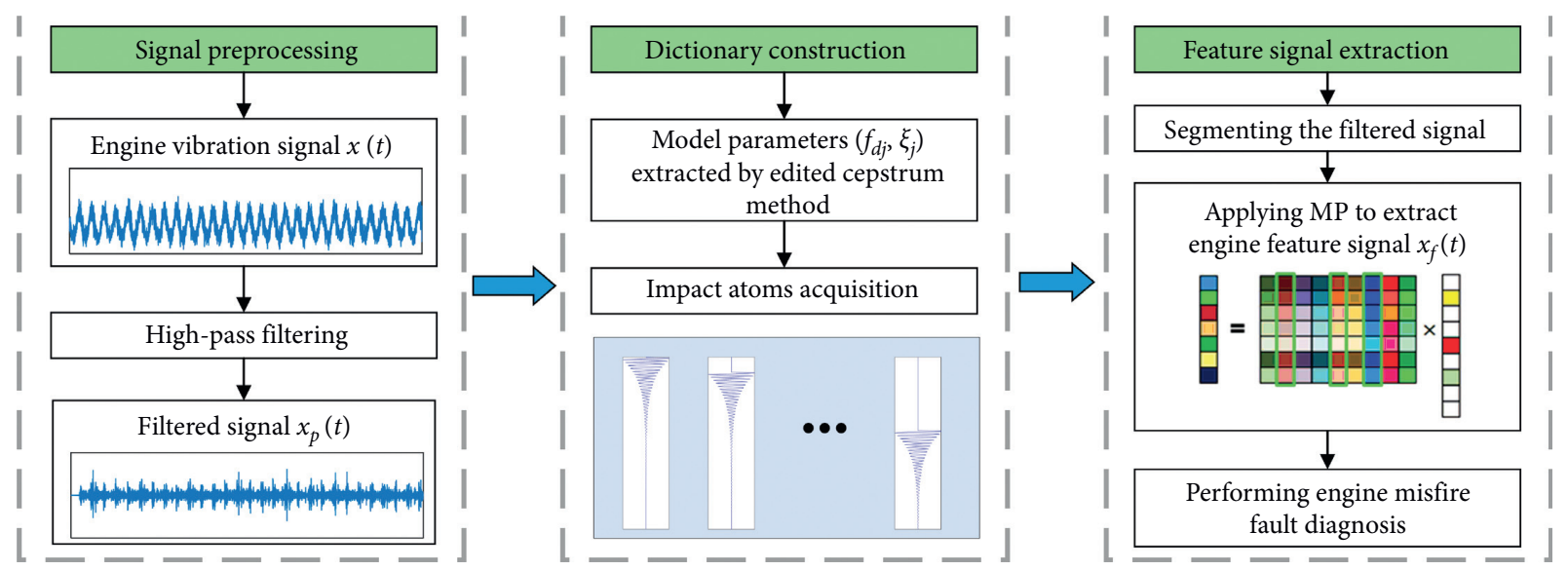

FIgURE 1: Flow chart of the engine signal feature extraction.

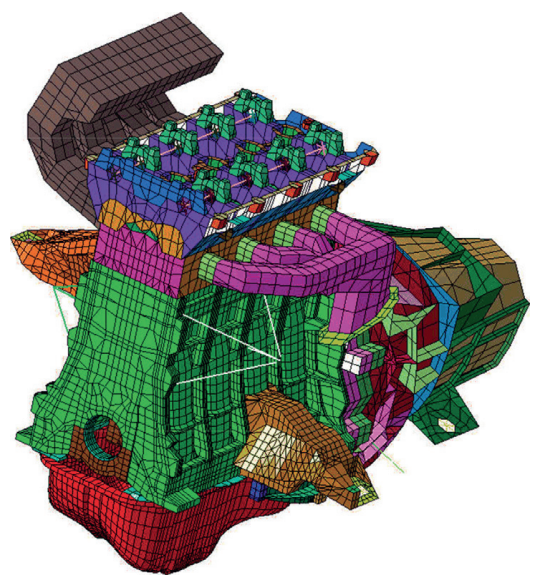

(a)

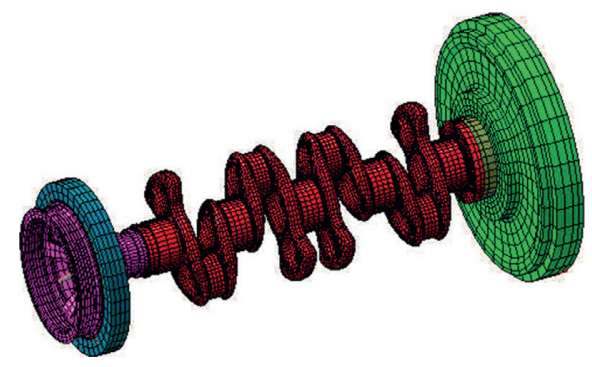

(b)

FIgURE 2: Finite element models. (a) Engine power unit. (b) Crankshaft.

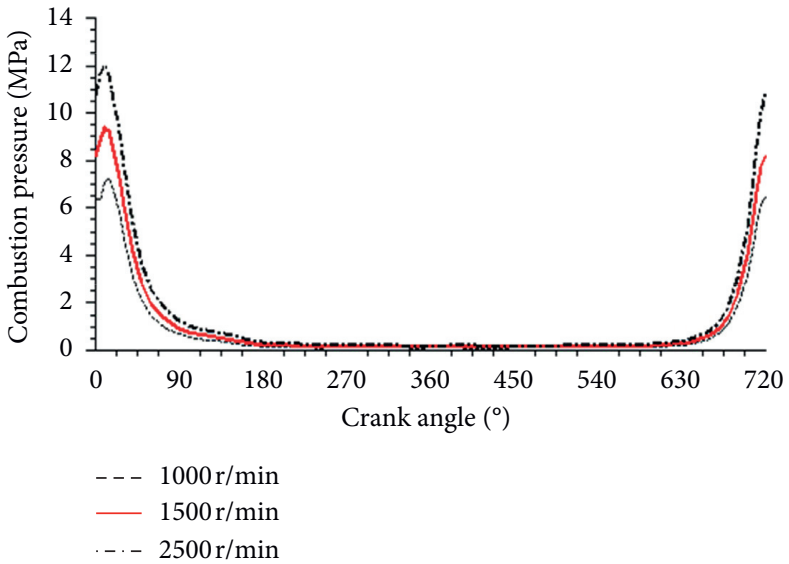

(a)

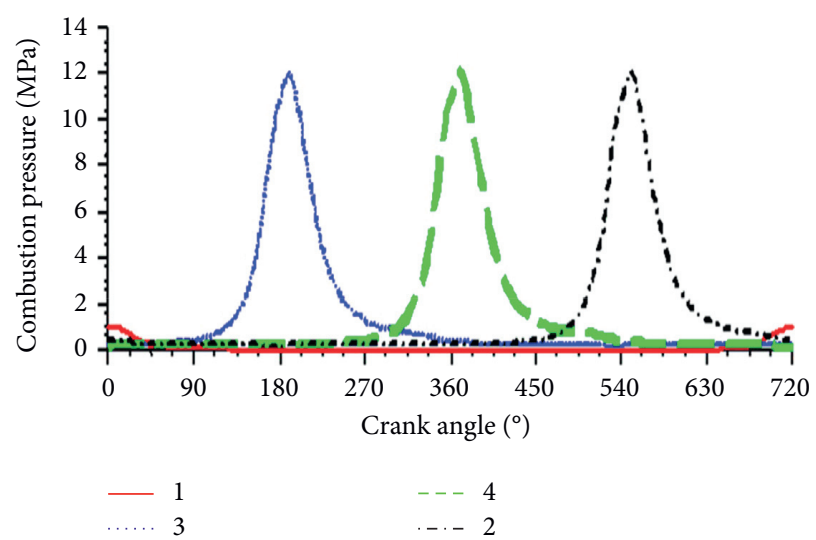

(b)

FIGURE 3: Combustion pressure variation along with crank angle. (a) Normal state. (b) First cylinder misfire fault in the four-cylinder engine model. 
angle represents the rotational angle of the crankshaft.

3.2. Normal State. Based on the dynamic model constructed in Section 3.1, the normal state signal is acquired on the hood with a sample frequency $f_{s}$ of $18748 \mathrm{~Hz}$. From Figure 4(a), it can be hardly seen of any features generated by engine impact forces both in the time domain and frequency domain. Even in the noiseless simulation condition, the features indicating the health state of the engine are still obscured by other low-frequency components. According to the signal analysis theory, impact forces will excite the system to vibrate at the high-frequency region. In order to eliminate the influence of low frequency as much as possible, combined with the spectrum of the original signal, a highpass filter with the cutoff frequency $f_{\text {cut }}=2000 \mathrm{~Hz}$ is applied. The filtered signal $x_{p}(t)$ is displayed in Figure $4(\mathrm{~b})$, where the SNR of impact signals is improved significantly.

Then, multiorder model parameters that highly correlated with the engine impact feature signal are identified to construct the sparse dictionary, and MP is further utilized to extract impact features from the filtered signal, and the iteration value $\delta$ is set as 0.001 , which could make the residual signal as small as enough and ensure the accuracy of the reconstructed signal according to Reference [14]. Here, the results are illustrated in Table 1 and Figure 5. Almost all identified frequencies from Table 1 are located at corresponding resonance regions in Figure 4(b), which demonstrates the correctness of the identification results and further provides high quality parameters for the dictionary design. As can be seen in Figure 5, nearly all the amplitudes and moments of the impact features in the extracted signal match well with those of the filtered noiseless signal. The results strongly verify the effectiveness of the proposed method in the feature extraction aspect. Moreover, each impact feature that is generated by the healthy engine cylinder is clearly exposed, which reveals the health state of the engine.

To further research the antinoise performance of the proposed method, a Gaussian white noise with $\mathrm{SNR}=5 \mathrm{~dB}$ is added into the simulation signal of Figure 4(a). The filter parameters are the same as the noiseless condition, and corresponding results are shown in Figure 6. Compared with Figure 4(b), the impact signals in Figure 6(b) are overwhelmed by noise after removing low-frequency interferences, which increases the difficulty in extracting the engine feature signal. Although, few extracted impact features exist slightly different, the vast majority of extracted signals obtained from the proposed method are still in accordance with the filtered noiseless signal, as shown in Figure 7. In sum, the proposed method can extract engine features well under low SNR conditions.

In order to further illustrate the advantage of the proposed method under low SNR, wavelet decomposition is chosen to process the same simulation signals in terms of its good feature extraction ability. In wavelet decomposition, $\mathrm{db} 10$ is selected as the basic function of the wavelet, and the decomposition layer is set as 4. FS represents the filtered signal; $\mathrm{d} 1, \mathrm{~d} 2, \mathrm{~d} 3$, and $\mathrm{d} 4$ denote the decomposition results. In the noiseless condition, it can be clearly seen that the wavelet decomposition method is able to extract engine impact features as effectively as the proposed method, as shown in Figure 8, when compared with the results in Figure 7 in the noise condition; though the wavelet decomposition method could denoise the filtered signal to some extent and make a few impact features clearer, the majority of the impact features are still submerged in strong noise, which reduces the diagnosis accuracy of engine misfire fault. In summary, the proposed method cannot only effectively extract and highlight engine features in the noiseless condition but also in the low SNR condition.

3.3. Single-Cylinder Misfire Fault. Engine misfire fault is mainly due to its cylinder failing to ignite properly, namely, under the unfired state. When the engine has misfire fault in the cylinder, corresponding impact signals will disappear theoretically due to its unsuccessful ignition in the faulty cylinder. Hence, the misfire fault feature can be described as a series of impact feature signal in the time domain; among the signals, some of the impact waves will miss due to the faulty cylinder.

Like the normal state, the single-cylinder misfire fault signal obtained from Section 3.1 also processed in the noiseless condition and $5 \mathrm{~dB}$ noise condition. As shown in Figure 9(a), it is still difficult to diagnose engine misfire fault by simply observing the original signal even in the noiseless condition. After removing interferences caused by lowfrequency components, the impact features have appeared out much more clearly in Figure 9(b). Moreover, it can be found that one impact feature is missing in the four consecutive impact intervals by comparing with the filtered signal under the normal state. This phenomenon just intuitively reveals the features of engine misfire fault and demonstrates the correctness of the simulation signal generation model in turn. Then, the feature signal extracted by the proposed method is illustrated in Figure 10, which shows a high similarity to the filtered signal, and its diagnosis features are also clearly exposed.

Under $5 \mathrm{~dB}$ noise condition, signals are more complex to analyse and basically hard to see any impact features both in the original signal and filtered signal, as shown in Figure 11. Hence, the proposed method is utilized to extract important impact features indicating engine misfire fault. From Figure 12 , all the engine ignition impact features with high amplitude are well extracted, and some weak impact features are also exposed slightly because of noise influence. In general, although the unavoidable noise coming from the environment may cause certain weak pseudoimpact features, the proposed method still can effectively extract engine misfire fault features with much higher amplitude to make correct diagnosis under the noise condition.

Similar to the normal state, the misfire signals are also performed by the wavelet decomposition method. As the results shown in Figure 13, engine misfire fault features can be well extracted under the noiseless condition. When it comes to the low SNR situation, majority of the impact 

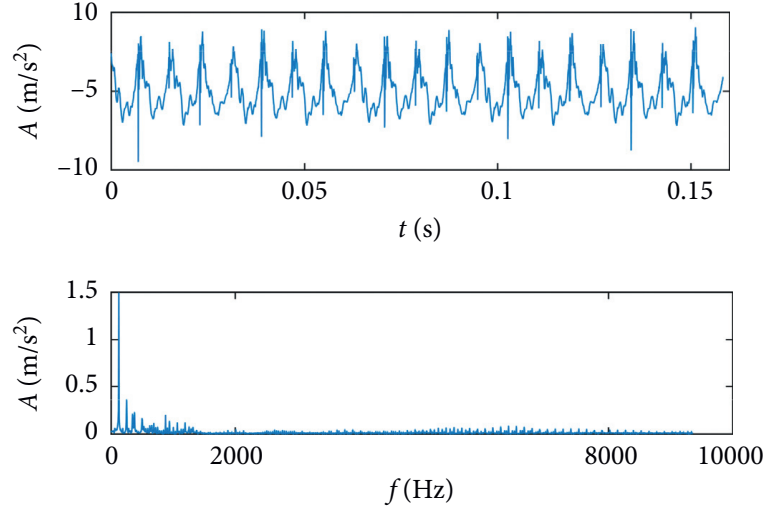

(a)
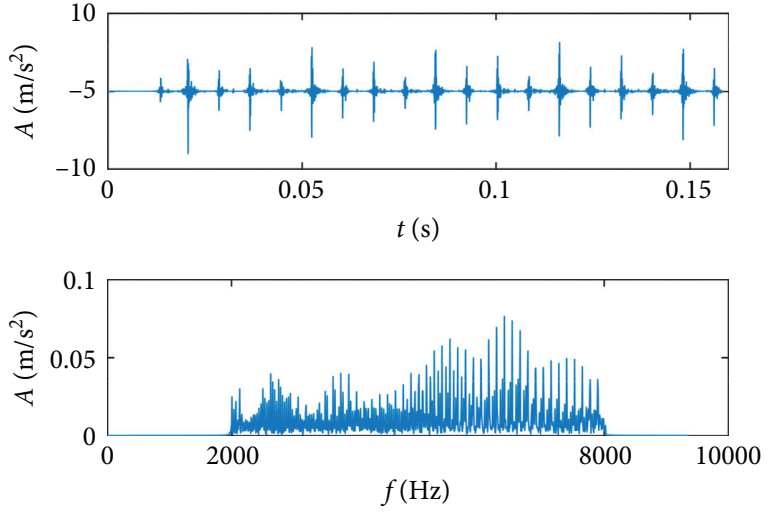

(b)

FIGURE 4: Vibration noiseless signal of the time domain and frequency domain under the normal state. (a) Original signal. (b) Filtered signal.

TABLE 1: The simulation results of multiorder model parameters' identification.

\begin{tabular}{lcccccc}
\hline Parameter & 1 & 2 & 3 & 4 & 5 & 6 \\
\hline $\begin{array}{l}\text { Frequency } f_{d j} \\
(\mathrm{~Hz})\end{array}$ & 2189 & 3996 & 5483 & 5969 & 7022 & 7901 \\
$\begin{array}{l}\text { Damping ratio } \\
\xi_{j}\end{array}$ & 0.0737 & 0.0286 & 0.0475 & 0.0095 & 0.0992 & 0.0125 \\
\hline
\end{tabular}

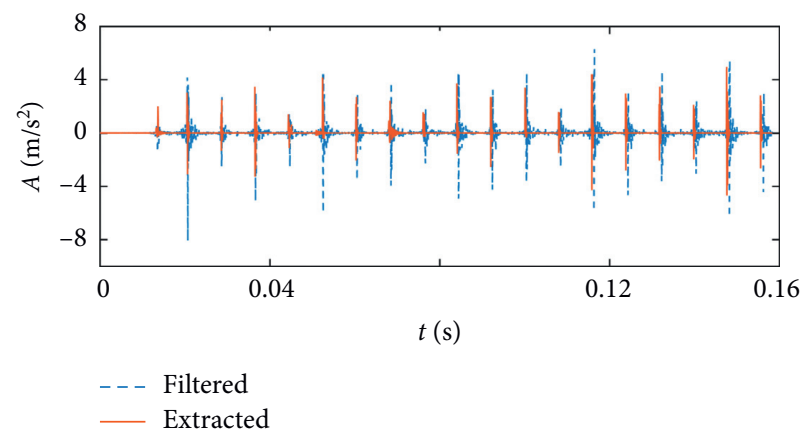

Figure 5: Filtered noiseless signal and extracted feature signal under the noiseless condition.

features are still covered by noise interferences, which hinders the diagnosis of engine misfire fault and further illustrates the superiority of the proposed method.

\section{Experiment Verification}

To further verify the effectiveness of the proposed method, a four-stroke in-line four-cylinder multipoint injection gasoline engine with a displacement of $1.6 \mathrm{~L}$ was operated under the normal state and single-cylinder misfire fault state, respectively. As shown in Figure 14, the vibration signal was obtained by the PCB vibration acceleration sensor mounted on the hood. The signal acquisition equipment contains the MKII signal collector and PAK acquisition and computer analysis system. The sampling frequency was set as $10240 \mathrm{~Hz}$. Misfire fault of the engine was accomplished by cutting off the corresponding cylinder fuel supply.
Additionally, the experiment was carried out under no load because of the limited conditions. Comparison analysis with the experimental signal illustrates the advantages of the proposed method in terms of feature extraction ability.

4.1. Normal State. As can be seen in Figure 15(a), the experimental signal acquired on the hood also includes lowfrequency components' interference, while the important high-frequency regions containing impact features are suppressed largely. This also verifies the correctness of the constructed dynamic model from another aspect by observing the similarity of simulation signals and experimental signals. Therefore, a high-pass filter is applied to highlight the impact features of the original signal as in simulation. The results are presented in Figure 15(b); compared with the unfiltered signal, the SNR of the filtered signal is indeed improved, and some weak impact features can be observed. However, it is still difficult to diagnose the health state of the engine by the current signal because of the complex noise interferences.

The multiorder model parameters affecting impact vibration characteristics are identified and listed in Table 2. The filtered signal is then utilized to exhibit the feature extraction superiority of the proposed method, as shown in Figure 16. Most of the impact features have been effectively extracted as well as the impact amplitudes. Compared with the filtered signal, the extracted signal is clearer and can better represent the engine operation condition. Furthermore, although there are few weak impact features influenced by noise, the amplitudes of most of the reconstructed signals are the same as those of the filtered signals and there is no periodic lowamplitude impact, which are consistent with the normal simulations. Hence, the engine health state can be concluded to the normal state, and the results of the experiment further verify the effectiveness of the proposed method.

4.2. Single-Cylinder Misfire Fault. The misfire fault experiment was also conducted on the same condition with the normal state. As illustrated in Figure 17(a), the original vibration signal obtained from the hood is disorderly and 


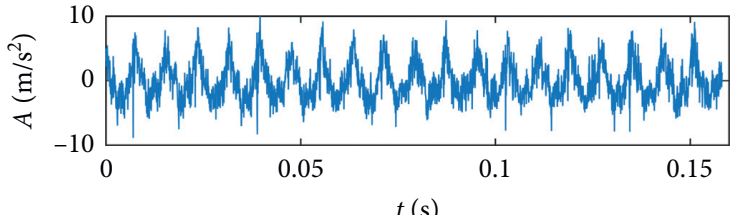

$t(\mathrm{~s})$

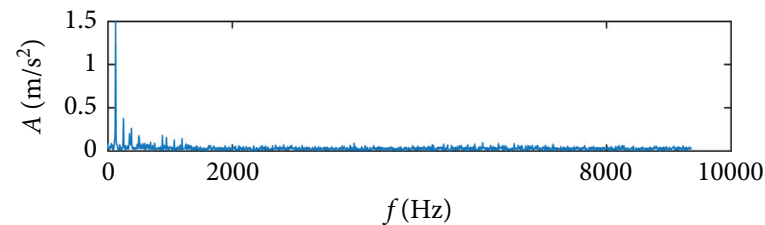

(a)
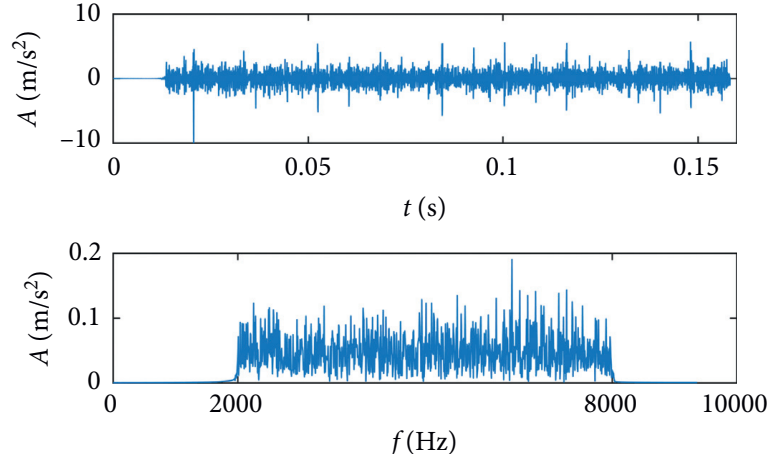

(b)

Figure 6: Vibration signal of the time domain and frequency domain under the normal state with $5 \mathrm{~dB}$ white noise. (a) Original signal. (b) Filtered signal.

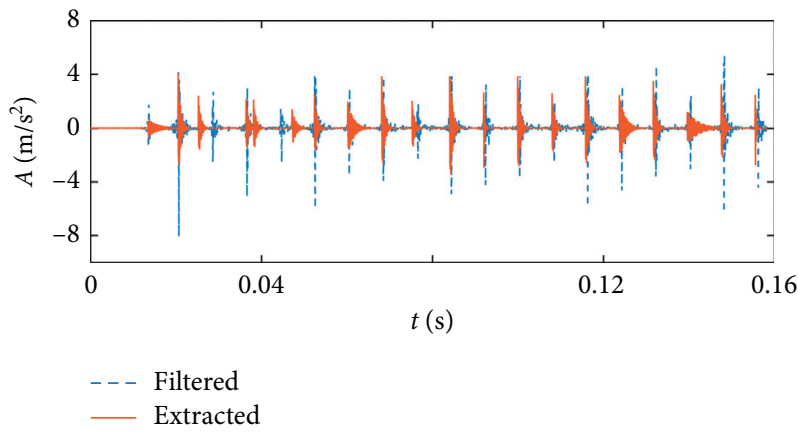

FIGURE 7: Filtered noiseless signal and extracted feature signal under $5 \mathrm{~dB}$ noise condition.

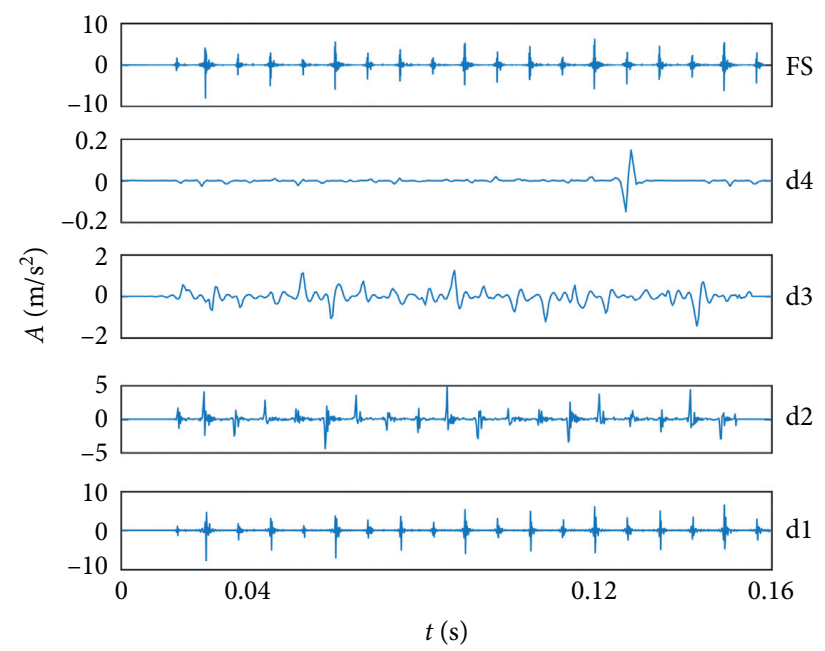

(a)

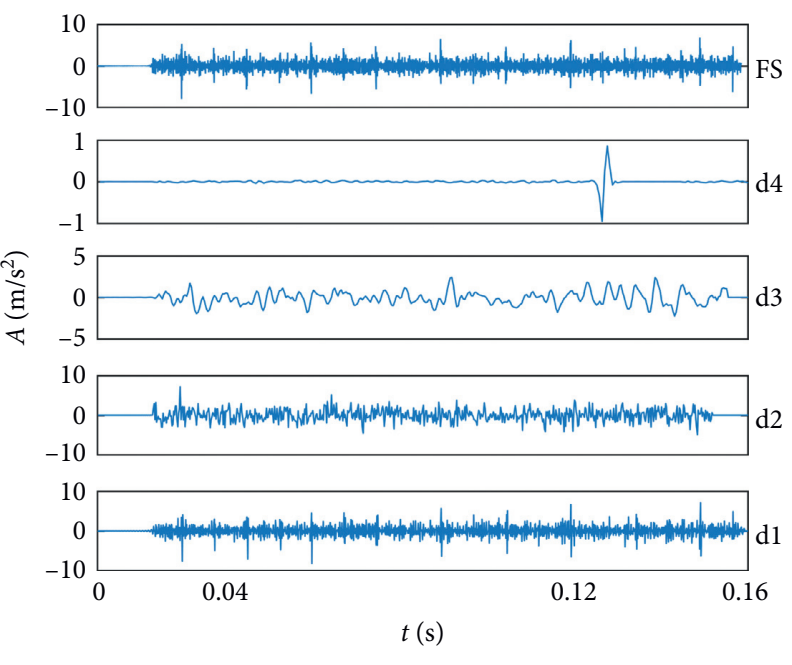

(b)

FIGURE 8: The results of the normal simulation signal processed by wavelet decomposition. (a) Noiseless. (b) Under $5 \mathrm{~dB}$ noise.

cannot be observed as useful information. After filtering the low-frequency noise, impact features located in the resonance area are highlighted, but still with some noise disturbances influencing engine health diagnosis.

Therefore, the impact features indicating the engine health state are further extracted by the proposed method. According to the misfire fault simulation results under noiseless and noise conditions, it can be known from that when single-cylinder misfire fault occurs, the extracted signal will still show equally spaced impacts rather than directly missing one impact. More importantly, the impact feature related with the faulty cylinder 


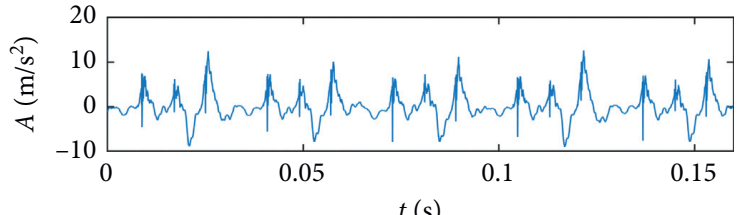

$t(s)$

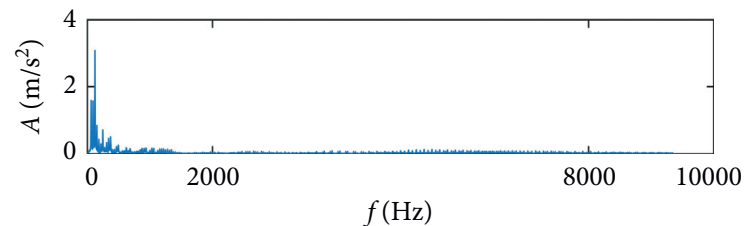

(a)

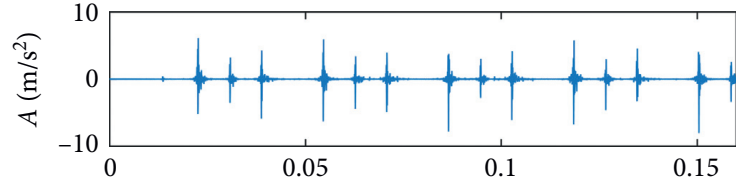

$t(\mathrm{~s})$

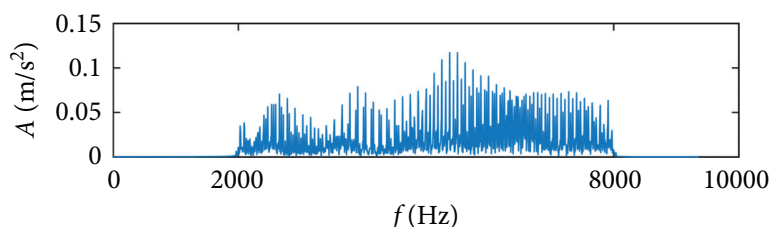

(b)

FIgURE 9: Vibration signal of the time domain and frequency domain under the misfire state. (a) Original signal. (b) Filtered signal.

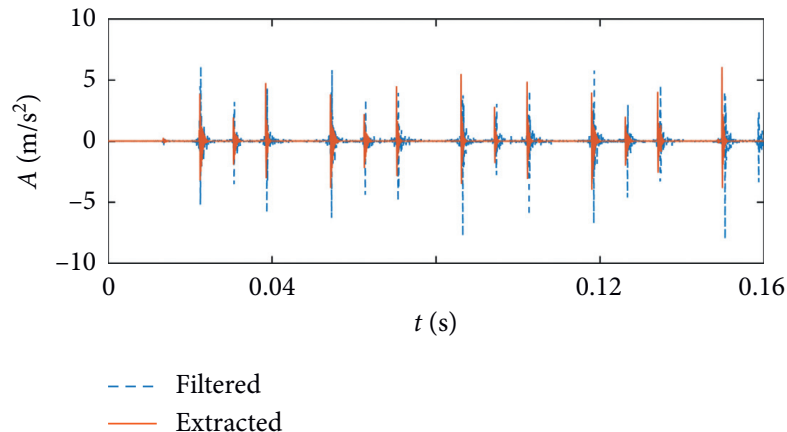

FIGURE 10: Filtered noiseless signal and extracted feature signal under the noiseless condition with single-cylinder misfire fault.

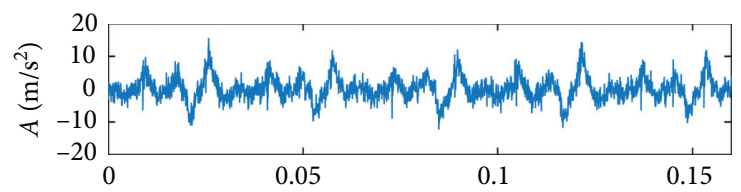

$t(\mathrm{~s})$

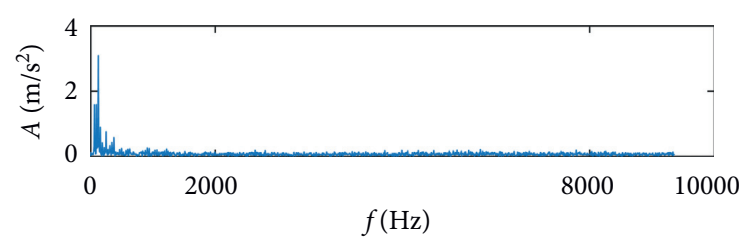

(a)
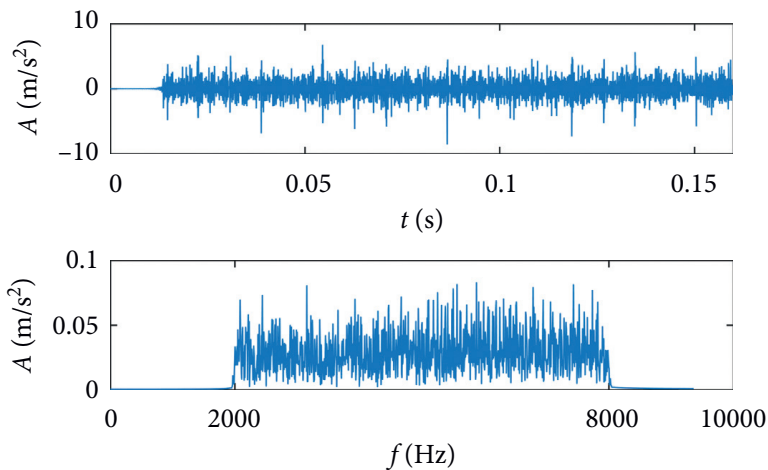

(b)

Figure 11: Vibration signal of the time domain and frequency domain under the misfire state with $5 \mathrm{~dB}$ white noise. (a) Original signal. (b) Filtered signal.

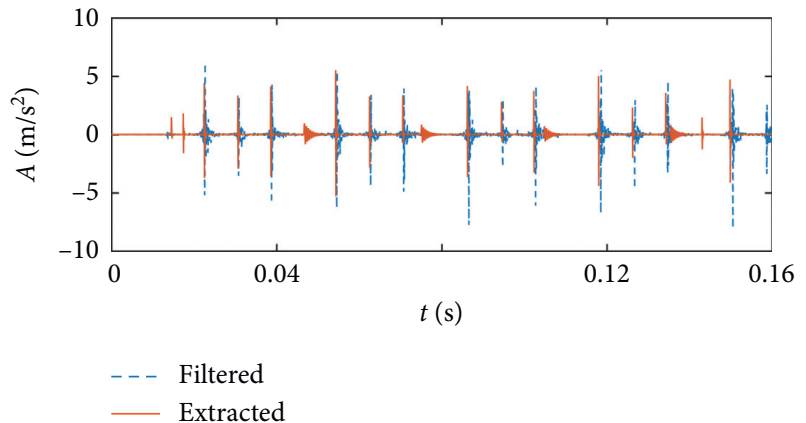

FiguRE 12: Filtered noiseless signal and extracted feature signal under $5 \mathrm{~dB}$ noise condition with single-cylinder misfire fault. 


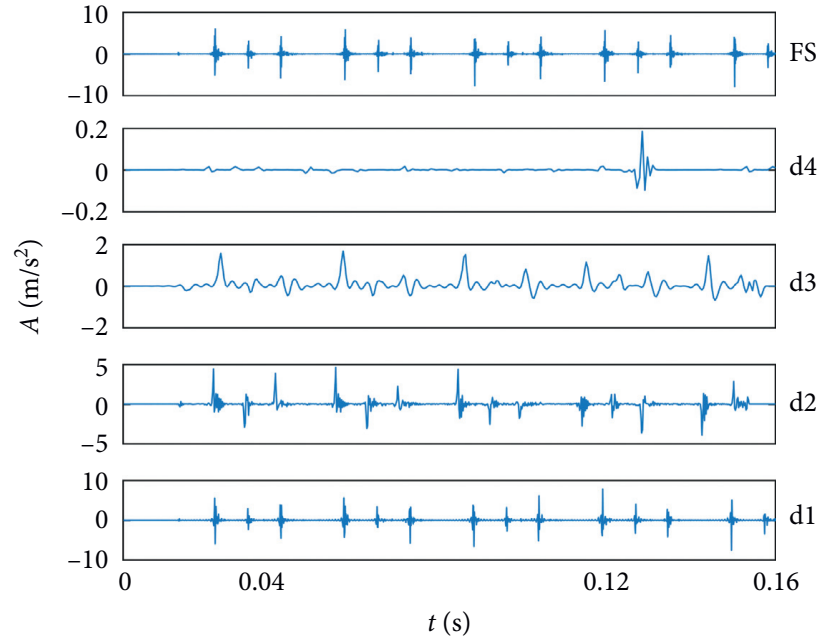

(a)

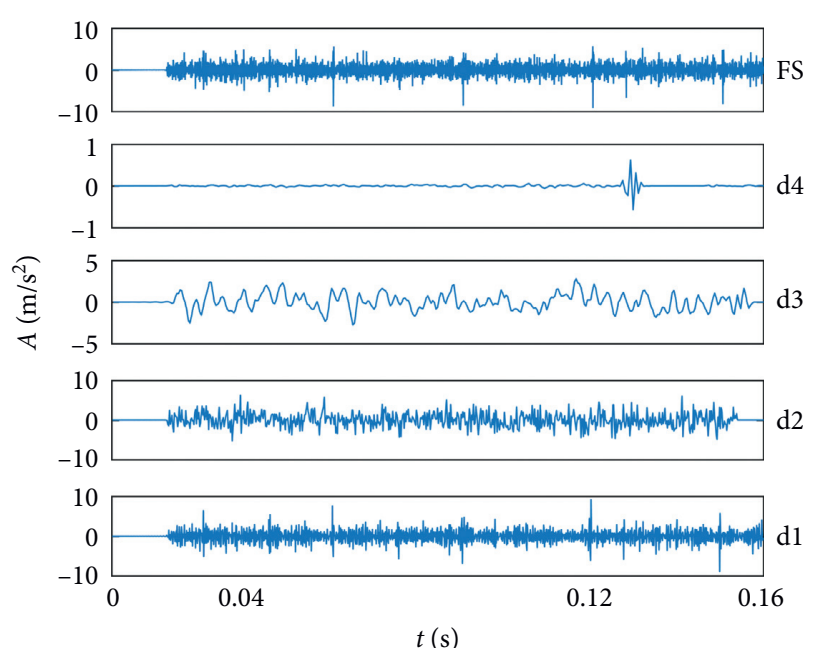

(b)

Figure 13: The results of the engine single-cylinder misfire fault signal processed by wavelet decomposition. (a) Noiseless. (b) Under $5 \mathrm{~dB}$ noise.

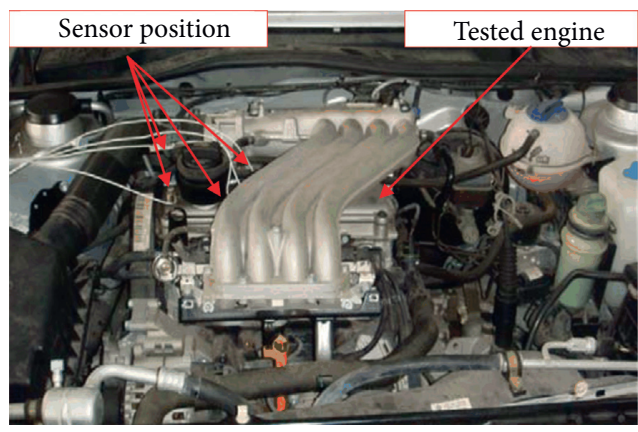

(a)

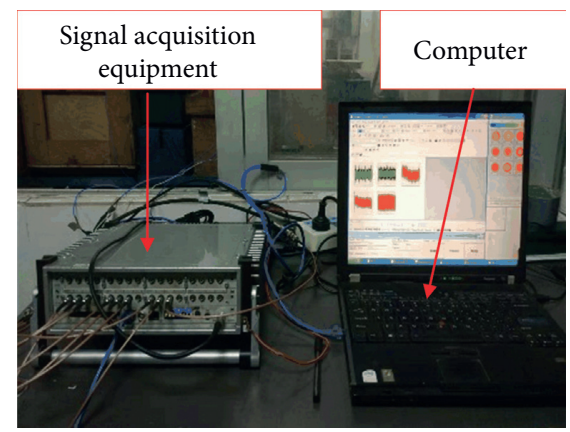

(b)

Figure 14: Experimental devices. (a) Tested engine. (b) Signal acquisition equipment.

TABLE 2: The experimental results of multiorder model parameters' identification.

\begin{tabular}{lcccc}
\hline Parameters & 1 & 2 & 3 & 4 \\
\hline Frequency $f_{d j}(\mathrm{~Hz})$ & 3089 & 3453 & 4099 & 4891 \\
Damping ratio $\xi_{j}$ & 0.0132 & 0.0416 & 0.1730 & 0.0150 \\
\hline
\end{tabular}

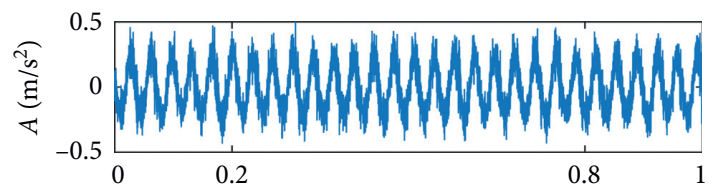

$t(\mathrm{~s})$

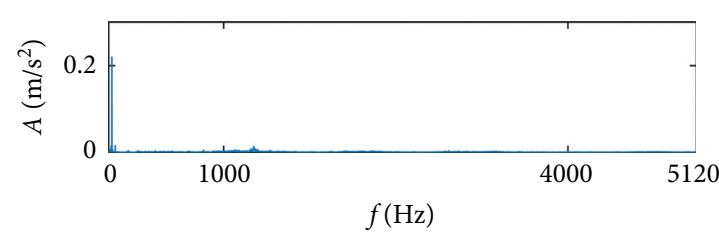

(a)

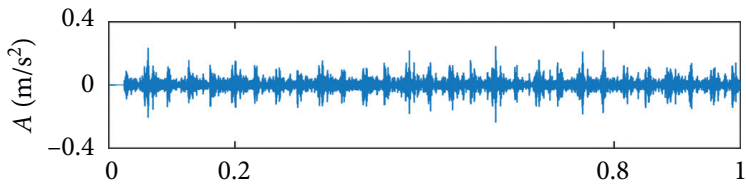

$t(\mathrm{~s})$

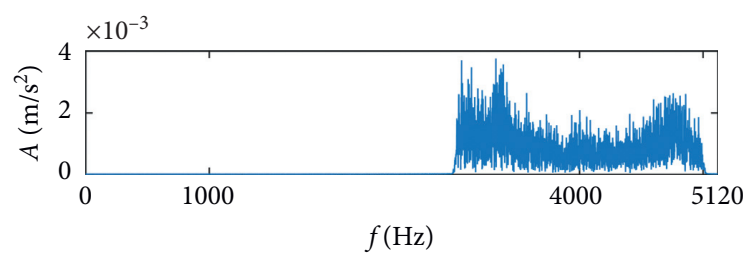

(b)

FIGURE 15: Experimental signal of the time domain and frequency domain under the normal state. (a) Original signal. (b) Filtered signal. 


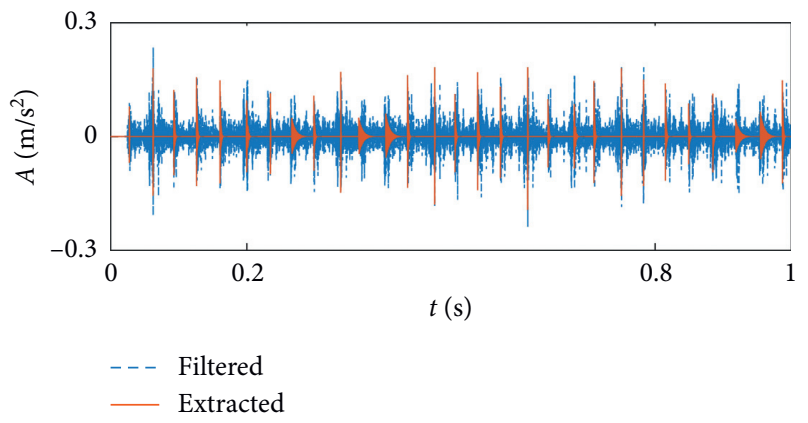

FIGURE 16: Experimental normal signal processed by the proposed method.
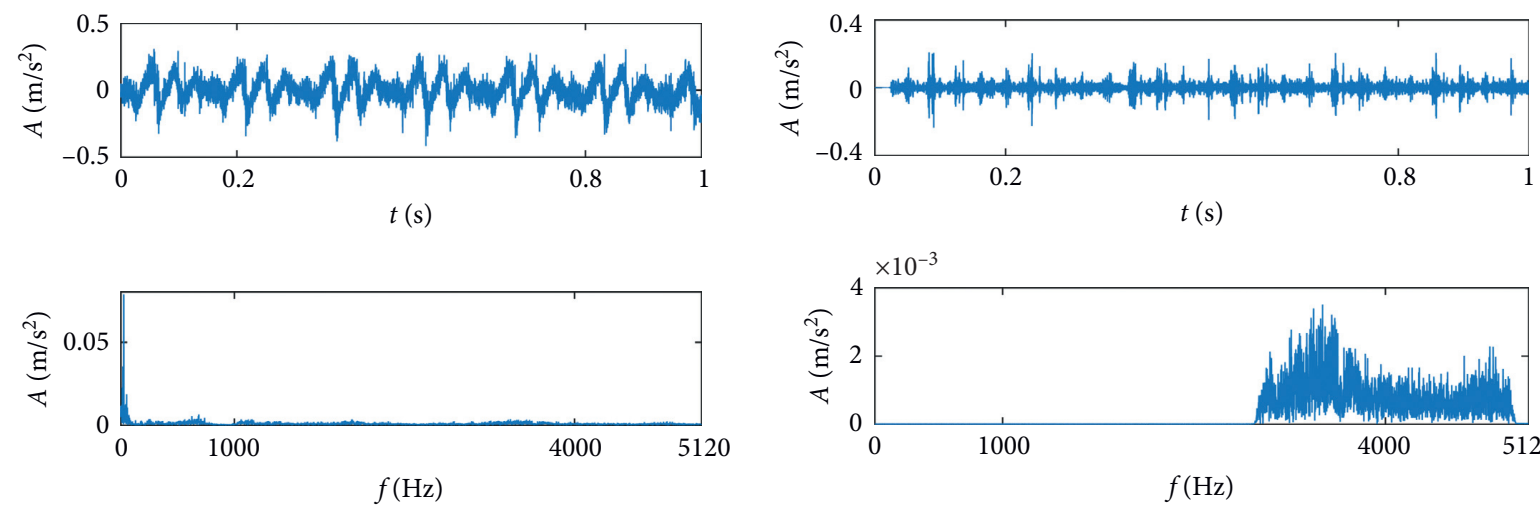

(a)

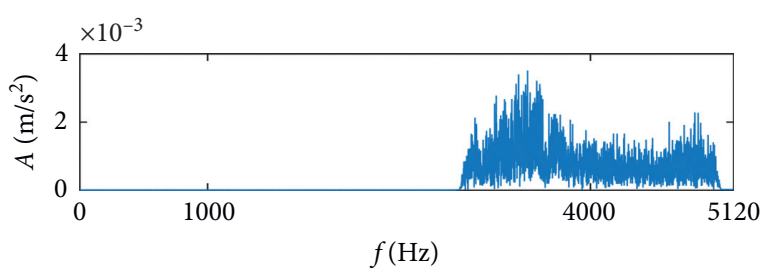

(b)

Figure 17: Experimental signal of the time domain and frequency domain under misfire fault. (a) Original signal. (b) Filtered signal.

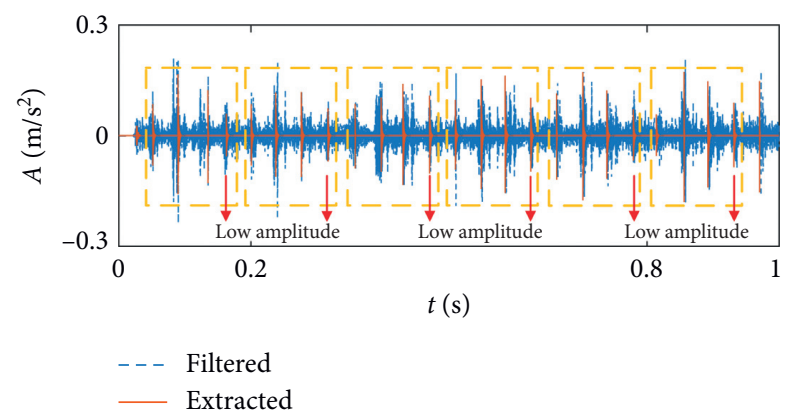

FIGURE 18: Experimental misfire fault signal processed by the proposed method.

has lower amplitude than others and shows quasi-periodicity. As shown in Figure 18, the results from the experimental signal are similar to the simulation results and therefore the engine misfire fault is diagnosed, which further verifies the correctness of the proposed method.
The results processed by wavelet decomposition are illustrated in Figure 19. It can be clearly seen that both extracted signals under the normal state and misfire state are submerged by strong noise, which hinders the engine misfire fault diagnosis. The comparison results further show the feature extraction advantage of the proposed method. 


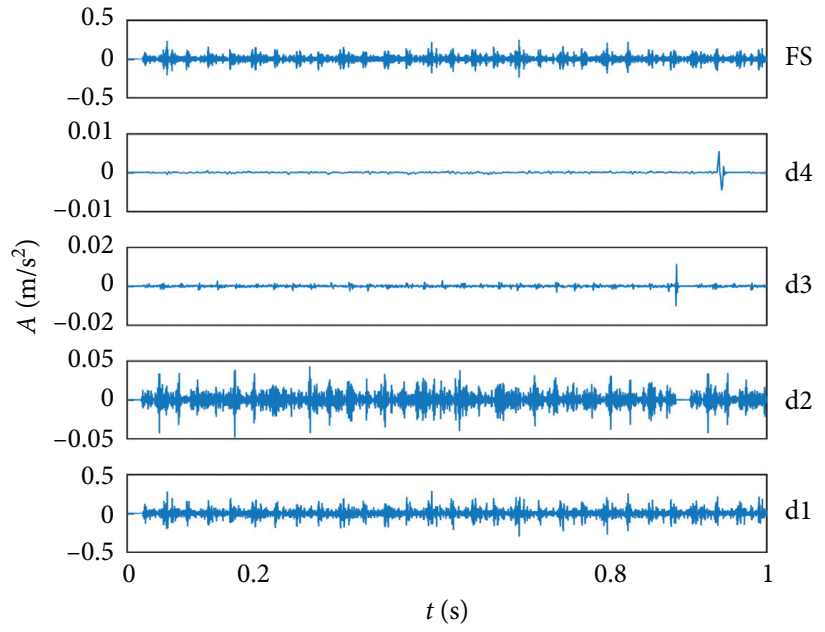

(a)

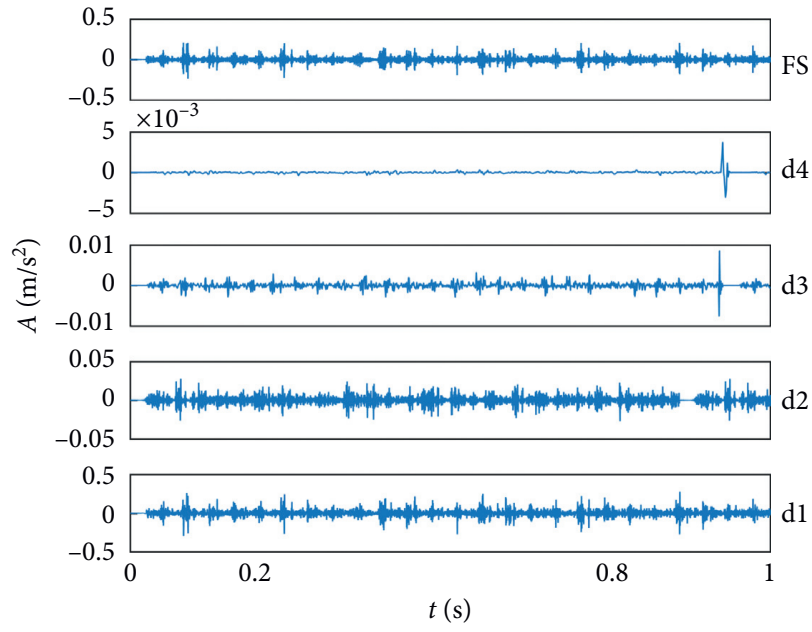

(b)

Figure 19: The results of the experimental signal processed by wavelet decomposition. (a) Normal state. (b) Single-cylinder misfire fault state.

\section{Conclusions}

Based on signal sparse decomposition theory, a feature extraction method is proposed to accurately extract impact features indicating the engine health state. The unit response function that reflects the system modal characteristic is used as the atom to construct the sparse dictionary with clear physical meaning. Moreover, the segmental MP algorithm is applied to reconstruct the impact features from the original signal. Simulations and experimental tests both verify the effectiveness of the proposed method. Some important conclusions are as follows:

(1) A four-cylinder engine multibody dynamic model constructed by the AVL EXCITE platform can generate more realistic signals than oversimplified numerical simulation. Furthermore, it cannot only simulate the engine normal signal but also the engine misfire fault signal by adjusting the combustion pressure of the corresponding cylinder.

(2) Original simulation signals contain many noise interferences, in which exists low-frequency components generated by other rotational parts. In the normal state, the proposed method can effectively extract all impact features in the noiseless condition. Moreover, under the low SNR condition, it can still make submerged impact features exposed clearly.

(3) In the engine misfire fault state, the faulty cylinder will not produce impact features in the corresponding ignition moment theoretically. Hence, impact features can be well extracted but with missing one impact by the proposed method in the noiseless condition. However, in the low SNR condition, besides the normal state impact features can be extracted, the pseudoimpacts with lower amplitude also appeared out due to the noise interferences.
(4) In real automotive engine tests, the proposed method still makes full use of the fault information that is buried in the original signal and highlights impact features to accurately diagnosis the health state of the engine, especially detect engine misfire fault.

(5) Though the wavelet decomposition method could obtain good extraction results under the noiseless condition as the proposed method, when it comes to the low SNR condition, only the proposed method can effectively extract the feature signals that indicate the engine health state.

\section{Data Availability}

The primary data used to support the findings of this study are available from the corresponding author upon request.

\section{Conflicts of Interest}

The authors declare that they have no conflicts of interest.

\section{Acknowledgments}

This work was partly supported by the Natural Science Foundation of Guangdong Province, China (nos. 2018A030313947, 2019A1515011779, and 2020A1515010972), Fundamental Research Funds for the Central Universities (no. 2019MS054), and Key Project of Guangzhou Science and Technology Program, China (no. 201904010387).

\section{References}

[1] Y. Xu, B. Huang, Y. Yun, R. Cattley, F. Gu, and A. D. Ball, "Model based IAS analysis for fault detection and diagnosis of IC engine powertrains," Energies, vol. 13, no. 3, p. 565, 2020.

[2] C. Gu, X. Qiao, Y. Jin, and Y. Liu, "A novel fault diagnosis method for diesel engine based on MVMD and band energy," 
Shock and Vibration, vol. 2020, Article ID 8247194, 17 pages, 2020.

[3] N. Dayong, J. Yuhua, S. Hongyu et al., "Separation method of impulsive fault component for gasoline engine based on acoustic signal analysis," Shock and Vibration, vol. 2019, Article ID 8573479, 15 pages, 2019.

[4] F. Jiang, K. Ding, G. He et al., "Vibration fault features of planetary gear train with cracks under time-varying flexible transfer functions," Mechanism and Machine Theory, vol. 158, Article ID 104237, 2021.

[5] X. Bi, S. Cao, and D. Zhang, "Diesel engine valve clearance fault diagnosis based on improved variational mode decomposition and bispectrum," Energies, vol. 12, no. 4, p. 661, 2019.

[6] K. Vernekar, H. Kumar, and K. V. Gangadharan, "Engine gearbox fault diagnosis using empirical mode decomposition method and Naïve Bayes algorithm," Sādhanāa, vol. 42, no. 7, pp. 1143-1153, 2017.

[7] T. Figlus, Š. Liščák, A. Wilk, and B. Łazarz, "Condition monitoring of engine timing system by using wavelet packet decomposition of a acoustic signal," Journal of Mechanical Science and Technology, vol. 28, no. 5, pp. 1663-1671, 2014.

[8] W. Deng, H. Liu, J. Xu, H. Zhao, and Y. Song, “An improved quantum-inspired differential evolution algorithm for deep belief network," IEEE Transactions on Instrumentation and Measurement, vol. 69, no. 10, p. 7319, 2020.

[9] C. Du, W. Li, F. Yu et al., "Misfire fault diagnosis of automobile engine based on time domain vibration signal and probabilistic neural network," International Journal of Performability Engineering, vol. 16, no. 9, 2020.

[10] K. Jafarian, M. Mobin, R. Jafari-Marandi, and E. Rabiei, "Misfire and valve clearance faults detection in the combustion engines based on a multi-sensor vibration signal monitoring," Measurement, vol. 128, pp. 527-536, 2018.

[11] J. Tao, C. Qin, W. Li, and C. Liu, "Intelligent fault diagnosis of diesel engines via extreme gradient boosting and high-accuracy time-frequency information of vibration signals," Sensors, vol. 19, no. 15, p. 3280, 2019.

[12] Y. Liu, X. Chen, R. K. Ward, and Z. Jane Wang, "Image fusion with convolutional sparse representation," IEEE Signal Processing Letters, vol. 23, no. 12, pp. 1882-1886, 2016.

[13] C. A. Riofrío, D. Gross, S. T. Flammia et al., "Experimental quantum compressed sensing for a seven-qubit system," Nature Communications, vol. 8, no. 1, pp. 1-8, 2017.

[14] G. He, K. Ding, and H. Lin, "Fault feature extraction of rolling element bearings using sparse representation," Journal of Sound and Vibration, vol. 366, pp. 514-527, 2016.

[15] F. Jiang, K. Ding, G. He et al., "Sparse dictionary design based on edited cepstrum and its application in rolling bearing fault diagnosis," Journal of Sound and Vibration, vol. 490, Article ID 115704, 2021.

[16] Y. Liu, J. Zhang, and L. Ma, “A fault diagnosis approach for diesel engines based on self-adaptive WVD, improved FCBF and PECOC-RVM," Neurocomputing, vol. 177, pp. 600-611, 2016.

[17] S. Li, Y. Zhang, L. Wang et al., "A CEEMD method for diesel engine misfire fault diagnosis based on vibration signals." in Proceeding of the 39th Chinese Control Conference (CCC), pp. 6572-6577, Shenyang, China, July 2020.

[18] T. Parikyan, T. Resch, and H. H. Priebsch, "Structured model of crankshaft in the simulation of engine dynamics with AVL/ excite," Internal combustion engine division fall technical conference, vol. 80159, pp. 105-114, 2001. 